\title{
Contenance, meine Damen und Herren, Contenance!
}

Die florierenden Zeiten sind offensichtlich vorüber, für das gesamte Land und seine Wirtschaft, ebenso aber auch für seine Ärzteschaft. Ein durchschnittlicher Realeinkommensverlust von fast 30\% in den letzten 25 Jahren, ein zunehmend durch Fremdbestimmung verengtes, bürokratisiertes Berufsfeld, eine Aus-, Weiter- und teilweise auch Fortbildung, die in einer immer gravierenderen Dauerkrise stecken, geben diesem düsteren Bild mehr als genügend Konturen.

Und nun TARMED. Was vielen über Jahre ein Hoffnungsschimmer war, scheint sich als Feuerschweif der Hölle zu entpuppen. Der Tarif ist vielen zu kompliziert, teilweise inkohärent und scheint entgegen den Versprechungen keinen Einkommensgewinn zu bringen. Man fühlt sich belogen, getäuscht und in die Irre geführt.

Vor allem das Gefühl, belogen worden zu sein, wiegt schwer. So schwer, dass es noch schwererfällt, rational dagegen zu argumentieren. Rationale Argumente gäbe es genug:

- Vieles wurde nicht so gesagt oder geschrieben, wie nun kolportiert. So wurde zugesichert und realisiert, dass sich die Einkommensdifferenzen vermindern würden. Der ewige «Dank» der Betroffenen ist uns gewiss;

- eine andere Zusicherung wäre auch verwegen gewesen, weil die absolute Einkommenshöhe von der allgemeinen Lohn-/Preissituation bzw. der wirtschaftlichen Lage abhängt. Und dies hat sich seit Beginn des Projektes dramatisch geändert - nicht zum Vorteil der Ärzteschaft;

- ein transparenter Tarif, der auf einer Minutage-Qualitäts-Matrix aufbaut und alle Leistungen umfasst, muss zwangsläufig komplex sein, aber nicht so komplex, dass ihn ein durchschnittlicher Arzt nicht für die tägliche Anwendung erlernen könnte.

Das aktuelle Malaise hat denn wohl auch tiefere Gründe:

1. die Ärzteschaft steht als Ganzes seit Jahren unter einem Trommelfeuer von Kritiken und Anwürfen durch Medien, Politik u.a.m.;

2. sinkende Realeinkommen, unattraktive Ausund Weiterbildung wie auch beengtes Berufsfeld sind Realitäten, die sich in den nächsten
Jahren noch verschärfen werden, kombiniert mit Medizin im freien Markt und im Rahmen eines globalisierten Dienstleistungsmarktes in Gestalt einer KVG-Revision Marke Couchepin.

Und nun auch einer der weniger Hoffnungsträger ein missgebildeter Flop. Was nun?

- Von Ärzten darf man erwarten, dass sie auch in Krisenzeiten Augenmass und ruhig Blut bewahren. So auch TARMED gegenüber. Sie werden dann entdecken, dass vermeintliche Fehlentwicklungen gar nicht real sind und sich auf die Defekte konzentrieren, die diskussionslos verhindert und eliminiert werden müssen. Zum Beispiel unsinnige Schnellschüsse eines BSV in Sachen Verrechnung Blutentnahme, nicht vereinbarungsgemässe Handhabung der Notfallpositionen.

- Lösung aber auch der seit Jahren anstehenden Probleme wie adäquate Tarifierung interventioneller, vor allem chirurgischer Leistungen und des schon nahezu den Albtraum von TARMED bildenden Belegarztproblems - bei dem sich aber auch gezeigt hat, dass beharrliches Insistieren zum Erfolg führt (wie an anderem Ort zu zeigen sein wird).

- Die Berufsorganisationen müssen sich hier an der Nase nehmen, die notwendigen Vorkehrungen zur Information der Basis ergriffen zu haben; sie haben gelernt und deshalb nun das Büro TARMED geschaffen.

- Erkennen der gravierenden Bedrohungen: die für die Ärzteschaft im Kern bedrohliche KVG-Revision. Ihre Protagonisten warten nur darauf, dass die Ärzteschaft 2004 mit TARMED scheitert, um dann 2005 die Argumentationsbasis für die Aufhebung jeglicher Vertragsregulation zu haben.

- Erkennen, dass die Leitungsgremien der Ärzteschaft nicht eine Bande von Idioten und Lügnern sind, sondern Menschen, die nach bestem Wissen und Gewissen ihre Arbeit tun, Fehler begehen, zweifelsohne, für diese kritisiert werden sollen, diskussionslos, aber nicht einfach fachlich und menschlich degradiert werden dürfen. 
Contenance hiess dies früher einmal, die Fähigkeit zur kritischen Mitwirkung in seiner Gesellschaft, verbunden mit Selbstkontrolle und Respekt vor dem anderen. Contenance wäre uns zu wünschen. Würden wir sie verlieren, so würden wir auch unser Recht verwirken, ein respektierter Beruf zu sein.

Hans Heinrich Brunner, Präsident FMH

\section{La maîtrise de soi}

L'âge d'or semble avoir perdu de son éclat, dans le pays tout entier comme pour son économie, et donc pour notre corps médical. Une perte de revenu réel de près de $30 \%$ en moyenne ce dernier quart de siècle; un champ d'action professionnel devenu manipulé, étroit, bureaucratisé; une formation prégraduée, postgraduée et même continue en crise durable: tout cela contribue à donner l'image assez sombre d'une crise qui semble durable.

Les affres du TARMED viennent s'y ajouter. Ce qui apparaissait pendant longtemps comme une lueur d'espoir s'est transformé en flammes infernales. Pour beaucoup d'entre nous, le tarif est trop compliqué, en partie incohérent et ne semble pas répondre à ses promesses en termes de revenus. On se sent floué, trompé, mené en bateau.

Ce sentiment d'avoir été trompé est difficilement supportable. Si difficilement supportable, d'ailleurs, que l'on peine à argumenter de manière rationnelle contre cette mauvaise farce. Les arguments rationnels ne manquent pourtant pas:

- Beaucoup d'éléments qu'on nous rapporte aujourd'hui ne correspondent pas à ce qui avait été dit ou écrit. Ainsi, une diminution des différences de revenus avait été prévue et garantie. La reconnaissance «éternelle» des intéressés nous est acquise!

- Donner d'autres garanties aurait été hardi, car la hauteur des revenus dépend de la situation générale des salaires et des prix à la consommation, donc de la situation économique. Celle-ci s'est considérablement dégradée depuis le début du projet, et pas en faveur du corps médical.
- Un tarif transparent fondé sur le minutage et la qualité et portant sur l'ensemble des prestations ne peut qu'être complexe, mais pas au point d'échapper à l'entendement d'un médecin de type courant dans son usage quotidien.

Le malaise actuel a des racines également plus profondes:

1. Depuis des années, le corps médical est sous le feu des critiques et des accusations de la part des médias et des politiques, pour ne citer qu'eux.

2. Les revenus réels en baisse, la perte d'attrait des études et de la formation postgraduée, le rétrécissement du champ professionnel sont des réalités qui ne feront que s'accentuer, en combinaison avec une médecine de libre marché dans le cadre d'un marché des prestations globalisé, dans l'esprit d'une révision de la LAMal à la Couchepin.

Et voici qu'en plus, un des rares projets porteurs d'espoir débouche sur un échec lamentable. Comment réagir?

- Même en temps de crise, on est en droit d'attendre des médecins qu'ils gardent leur sangfroid et le sens de la mesure. Ils feront bien d'adopter cette attitude aussi dans le cas du TARMED. Remarquant alors que certains prétendus développements erronés n'en sont pas, ils se concentreront sur les défauts réels qu'il s'agit indiscutablement de corriger et d'éliminer. Nous pensons par exemple aux actions précipitées et absurdes d'un OFAS en 
matière de facturation de prises de sang ou au traitement, non conforme aux conventions, des positions tarifaires applicables en cas d'urgence.

- D'autres problèmes perdurant depuis des années appellent eux aussi une solution rapide: les prestations de type interventionnel, avant tout chirurgical, qui doivent enfin faire l'objet d'une tarification adéquate et la question de la rémunération des médecins agréés, en passe de devenir le cauchemar du TARMED... des domaines où il est par ailleurs avéré qu'une insistance tenace peut conduire au succès (comme cela sera démontré dans un autre contexte).

- Les organisations professionnelles ont à se reprocher de n'avoir pas pris les mesures nécessaires pour informer la base. Tirant la leçon de ce manquement, elles viennent de créer le Bureau TARMED.

- Il importe de cerner à temps les menaces graves, voire existentielles, qui pèsent sur nous, telle la révision de la LAMal. Ses défenseurs n'attendent qu'un échec du corps médical dans l'introduction du TARMED en 2004 pour leur fournir, en 2005, des arguments en faveur de la suppression de toute réglementation contractuelle.

- Il convient, enfin, que d'aucuns reconnaissent que les organes directeurs du corps médical ne sont pas une bande de demeurés et de menteurs, mais des gens qui font leur travail au plus près de leur conscience, qui commettent certes des erreurs et doivent sans aucun doute être critiqués en conséquence, mais qu'il n'est tout simplement pas permis de rabaisser et d'humilier tant sur le plan humain que professionnel.

La maîtrise de soi: c'est ainsi qu'on dénommait autrefois la faculté d'agir au sein de la société en faisant preuve tout à la fois d'esprit critique, de retenue et de respect des autres... une qualité qui serait maintenant de mise et que je nous souhaite à tous! Si nous perdions cette maîtrise, nous perdrions en même temps tout droit à faire partie d'une profession respectée.

Hans Heinrich Brunner, président de la FMH 